\title{
Dirac nodal line in bilayer borophene: Tight-binding model and low-energy effective Hamiltonian
}

\author{
M. Nakhaee, ${ }^{1,2, *}$ S. A. Ketabi, ${ }^{2, \dagger}$ and F. M. Peeters ${ }^{1, \dagger}$ \\ ${ }^{1}$ Department of Physics, University of Antwerp, Groenenborgerlaan 171, B-2020 Antwerp, Belgium \\ ${ }^{2}$ School of Physics, Damghan University, P.O. Box 36716-41167, Damghan, Iran
}

(Received 12 April 2018; revised manuscript received 17 June 2018; published 6 September 2018)

\begin{abstract}
Bilayer hexagonal borophene, which is bound together through pillars, is a novel topological semimetal. Using density functional theory, we investigate its electronic band structure and show that it is a Dirac material which exhibits a nodal line. A tight-binding model was constructed based on the Slater-Koster approach, which accurately models the electronic spectrum. We constructed an effective four-band model Hamiltonian to describe the spectrum near the nodal line. This Hamiltonian can be used as a new platform to study the new properties of nodal line semimetals. We found that the nodal line is created by edge states and is very robust against perturbations and impurities. Breaking symmetries can split the nodal line, but cannot open a gap.
\end{abstract}

DOI: 10.1103/PhysRevB.98.115413

\section{INTRODUCTION}

Newly discovered Dirac [1,2], Weyl [3-6], and nodal line semimetals (NLS) [7-9] have resulted in novel states of matter. NLSs can be considered as precursor states for other topological states intermediate between insulators and metals. The valence and conduction bands in topological semimetals touch at either discrete points or extended lines, forming respectively Dirac or Weyl semimetals and NLSs. Topologically nontrivial surface states are one of the characteristic features of topological insulators that are protected by time reversal symmetry. Band degeneracy points or lines in topological semimetals are also protected by symmetries and are thus robust against external perturbations. Recently, alongside the traditional topological insulators, topological semimetals have attracted tremendous research interest.

NLSs are observed in several bulk materials, such as body-centered orthorhombic $\mathrm{C}_{16}$ [10] and hcp alkali earth metals [11] and, recently, also in some two-dimensional (2D) materials [12] like black phosphorus under pressure [13]. It turns out that there are many materials showing these topological states, ranging from hyper-honeycomb lattices [14] and 3D-honeycomb lattices [15] to high-temperature superconductors $[16,17]$ and nearly flat drumhead-like surface states [18]. There are a number of reports of the existence of two concentric Dirac nodal loops centered around one point in reciprocal space [19] or a single nodal line as found in $\mathrm{CaP}_{3}$ [20], $\mathrm{Ca}_{3} \mathrm{P}_{2}$ [21], and (Tl, $\left.\mathrm{Pb}\right) \mathrm{TaSe}_{2}$ [22].

Several novel properties have been predicted for NLSs, including special collective modes [23] and unique Landau levels [24]. The nodal line structure can also be considered as a precursor state for different topological states. For instance, a NLS can be converted to Weyl nodes [25-28] by taking spinorbit coupling into account, or nodal rings can evolve into

\footnotetext{
*mohammad.nakhaee@uantwerpen.be

${ }^{\dagger}$ saketabi@du.ac.ir

francois.peeters@uantwerpen.be
}

Dirac nodes, converting the NLS into a topological insulator by opening a gap [29].

Two-dimensional (2D) materials have also attracted the interest of scientists because of the possibility of applications in high-speed nanodevices due to their special electronic properties. The realization of $2 \mathrm{D}$ topological semimetals will provide new platforms for the design of novel quantum devices at the nanoscale. In contrast to three-dimensional topological Dirac semimetals, the nodal lines in many 2D materials such as monolayer hexagonal lattices [30] and honeycomb-kagome lattices [12] are protected by mirror symmetry and require negligible spin orbit coupling [31]. Notwithstanding the lack of experimental realization of such structures up to now, there is still a need to predict new 2D materials with nodal lines that can be fabricated.

Recently, 2D boron sheets (called borophene) have been successfully synthesized on a silver crystal by vapor deposition [32-36]. The boron-based materials show different metallic [37], semimetallic [38], and semiconducting [39] behaviors. Boron atoms have four available orbitals with three occupied valence electrons, and because of electrondeficiency can form both covalent and ionic bonds. Various allotropes of boron-based structures can be formed such as clusters, nanotubes, 2D structures, and bulk materials [40-42]. Figure 2 shows the structure of bilayer borophene that are bound together by pillars. This structure, containing six atoms per primitive unit cell, is called 6B: $P 6 \mathrm{mmm}$, in which $P 6 \mathrm{mmm}$ stands for space group 191 included in the hexagonal crystal system. Using density functional theory (DFT) and a global minimum search optimization method we are able to investigate stable 2D bilayer borophene. Phonon dispersion calculations reveal that this structure is dynamically stable [43] and therefore has the potential to be successfully synthesised for practical applications. The hexagon unit cell includes six atoms, and the vectors $\mathbf{a}$ and $\mathbf{b}$ are the translational unit cell vectors that make a 120 degree angle. In the center of the unit cell there are two atoms that form a pillar between the two layers. Most importantly, the 6B:P6 $6 \mathrm{~mm}$ structure exhibits a Dirac nodal line with a Fermi velocity 


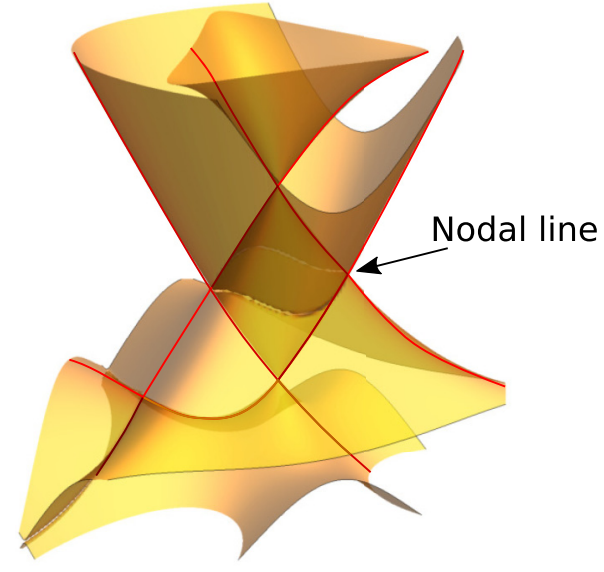

FIG. 1. 3D plot of the band structure of 6B:P6mmm calculated by DFT near the Fermi level around the $K$ point.

comparable to that of graphene. It should be noted that the authors of Ref. [43] only plotted the ban -structure along some specific $k$ paths, which is not sufficient to find out the shape of the energy surface in 3D. We plotted the band structure in 3D and found that the reported Dirac cones are only two points from a Dirac nodal line. Figure 1 illustrates the 3D plot of the band structure of 6B:P6mmm calculated by DFT near the Fermi level in the $K$ point.

Efforts to search for potentially stable boron-based sheets with low energy and novel electronic properties are of great interest. In this paper, we report on Dirac nodal line fermions in 6B: $P 6 \mathrm{mmm}$ borophene bilayer based on first principles calculations, and we construct a tight-binding model via the Slater and Koster (SK) method [44] to reproduce the band energies calculated by DFT. The SK scheme is a powerful method based on the linear combination of atomic orbitals (LCAO) method. The Dirac nodal line in 6B:P6mm forms a loop centered around the $K$ point in reciprocal space with dispersion in energy. Also we present a four-band effective low-energy Hamiltonian to describe the nodal line, which can be used as a platform to study the novel physical properties of such two-dimensional Dirac nodal semimetals. Our results provide opportunities to understand the nodal line structure and its corresponding surface states and can be used to realize high-speed spinless devices.

\section{ELECTRONIC STRUCTURE USING DFT}

The electronic properties of 6B:P6mm are investigated by first-principles calculations using the OPENMX package [45]. Atomic structure relaxations and calculations of electronic band-structure were performed within the linear combination of pseudo-atomic orbitals (LCPAO) method [46]. Structure relaxations were carried out with the thershold $10^{-5} \mathrm{eV} / \mathrm{A}$ for the maximum value of the forces between the boron atoms. The generalized gradient approximation was applied for the exchange-correlation energy using the Perdew, Burke, and Ernzerhof (PBE) functional [47,48] with a cutoff energy of $400 \mathrm{eV}$ for the plane-wave basis. Numerical integrations in the Brillioun zone were evaluated with the Monkhorst-Pack mesh $(12 \times 12 \times 1)$. (a)

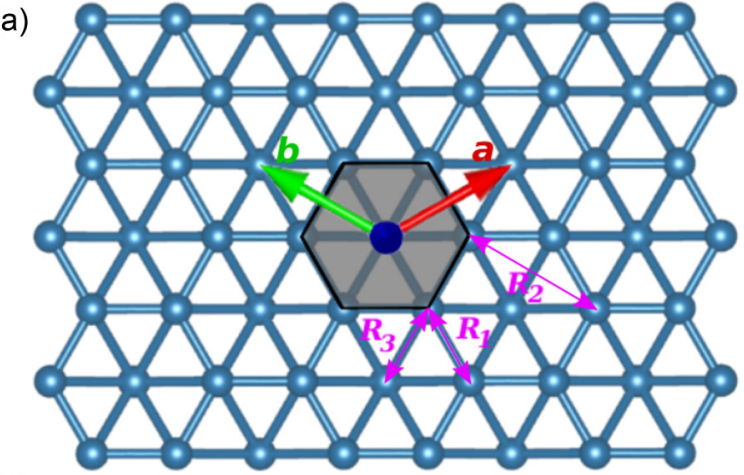

(b)

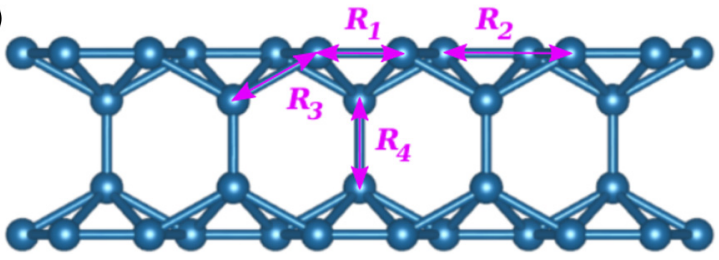

FIG. 2. Top (a) and side (b) views of the structure of bilayer borophene 6B: $P 6 \mathrm{mmm}$. The gray hexagon area is the primitive cell. The vectors $\mathbf{a}$ and $\mathbf{b}$ denote the translational vectors of the crystal lattice.

After structure relaxation the lattice constant was calculated as $|\mathbf{a}|=|\mathbf{b}|=2.85 \AA$ with $\theta_{\mathbf{a b}}=120^{\circ}$, which are in agreement with a recent work [43]. This structure includes two honeycomb lattices at the top and the bottom layers which are connected through pillars. Thus the two layers of bilayer borophene are chemically bound together, in contrast to bilayer graphene which is held together by only weak van der Waals forces. After optimizing the atomic positions, one finds the distances $R_{1}=1.65 \AA, R_{2}=2.85 \AA, R_{3}=1.89 \AA$, and $R_{4}=1.70 \AA$. The band structure of 6B:P6mmm is displayed in Fig. 3. The contributions of the orbitals $s, p_{x}, p_{y}$, and $p_{z}$ are shown by different colors. Blue and red circles illustrate the location of two Dirac cones, which are related to the electrons and holes, respectively. The nodal line is created by the intersection of two Dirac cones formed by the $p_{z}$ orbital centered at the point $K$ in the Brillouin zone.

\section{TIGHT-BINDING MODEL AND COMPARISON WITH DFT CALCULATIONS}

For future purposes we develop a tight-binding (TB) model to reproduce the DFT band energies. We include the potentials of atoms on each layer, which can be only sensed by the neighboring atoms in the same layer, and electrons can hop between layers only through the pillars. To construct a TB model we consider the following matrix elements [49]:

$$
\begin{aligned}
H_{i v, i^{\prime} v^{\prime}} & =\left\langle\phi_{\nu}\left(r-r_{i}\right)|H| \phi_{\nu^{\prime}}\left(r-r_{i^{\prime}}\right)\right\rangle, \\
S_{i v, i^{\prime} v^{\prime}} & =\left\langle\phi_{\nu}\left(r-r_{i}\right) \mid \phi_{\nu^{\prime}}\left(r-r_{i^{\prime}}\right)\right\rangle .
\end{aligned}
$$

Here $H$ is the single-electron Hamiltonian and $S$ is the overlap matrix resulting from the nonorthogonal basis functions [50-52]. The integrals are evaluated over the unit cell in real space and $i$ and $v$ run over all atoms in the unit cell and the orbitals $s, p_{x}, p_{y}$, and $p_{z}$, respectively. The eigenenergy 
TABLE I. The Slater-Koster parameters for 6B: $P 6 \mathrm{mmm}$. The $V$ parameters are in $\mathrm{eV}$ and the $S$ parameters are dimensionless.

\begin{tabular}{|c|c|c|c|c|c|c|c|c|}
\hline$R$ & $V_{s s \sigma}$ & $V_{s p \sigma}$ & $V_{p p \sigma}$ & $V_{p p \pi}$ & $S_{s s \sigma}$ & $S_{s p \sigma}$ & $S_{p p \sigma}$ & $S_{p p \pi}$ \\
\hline$R_{1}$ & -6.667 & 5.527 & 4.707 & -1.654 & 0.379 & -0.162 & 0.202 & 0.165 \\
\hline$R_{2}$ & 0.100 & 0.092 & -0.150 & -0.1968 & 0.035 & -0.039 & -0.035 & 0.062 \\
\hline$R_{4}$ & 4.560 & -1.446 & -0.738 & 4.189 & -0.193 & -0.054 & -0.017 & -0.047 \\
\hline
\end{tabular}

equation for a single electron can be written as follows:

$$
\sum_{v^{\prime}} \sum_{i^{\prime}}\left[H_{i v, i^{\prime} v^{\prime}}-\epsilon_{k} S_{i v, i^{\prime} v^{\prime}}\right] c_{i^{\prime} v^{\prime}}(k)=0 .
$$

The interatomic matrix elements are found by fitting the known band energies [53]. The matrix elements can be represented by the expectation values of the Hamiltonian in the basis of the directed orbitals in terms of eight integrals $\left(v_{s s \sigma}\right.$, $\left.v_{s p \sigma}, v_{p p \sigma}, v_{p p \pi}, s_{s s \sigma}, s_{s p \sigma}, s_{p p \sigma}, s_{p p \pi}\right)$ defined as follows:

$$
\begin{aligned}
\langle s|H| s\rangle & =v_{s s \sigma}, \\
\left\langle s|H| p_{i}\right\rangle & =n_{i} v_{s p \sigma}, \\
\left\langle p_{i}|H| p_{j}\right\rangle & =\left(\delta_{i j}-n_{i} n_{j}\right) v_{p p \pi}+n_{i} n_{j} v_{p p \sigma} .
\end{aligned}
$$

Note, one should use the rule of angular quantum number, $\left\langle l|H| l^{\prime}\right\rangle=(-1)^{l+l^{\prime}}\left\langle l^{\prime}|H| l\right\rangle$, to evaluate the complexconjugate hopping matrix elements. In Eq. (4) $n_{i}$ is the directional cosines defined by the following equation:

$$
n_{i}=\frac{\mathbf{r} \cdot \mathbf{e}_{i}}{|\mathbf{r}|}
$$

where $\mathbf{r}$ is the vector along the corresponding bond and $i$ runs over different Cartesian directions $(x, y$, and $z$ ). For the overlap matrix the corresponding expressions can be found by replacing $H$ by $S$ and $v$ by $s$ in Eq. (4). The unknown parameters are determined by a best fitting of the energy bands that are obtained by the DFT method. We calculate the values of the eight integrals up to distances of the three nearest-neighbor sites in each layer and for the pillars between layers.

6B: $P 6 \mathrm{mmm}$ lattice is a structure with a basis of six boron atoms. We assume a basis containing one atomic-like $s$ and three $p_{x}, p_{y}$, and $p_{z}$ orbitals per atom, which generates a band structure with 24 bands. However, we will concentrate on finding a satisfactory fitting for the first 11 bands. To calculate the TB Hamiltonian by using the eight Slater-Koster integrals, one needs the distances and the directional cosines in Eq. (4) for the different bonds, which we obtained from DFT. The distances between the boron atoms, $R_{i}$, corresponding to different types of bonds are shown in Fig. 2.

Using the Levenberg-Marquardt nonlinear fitting algorithm [54] we find the optimal Hamiltonian and overlap matrices. The Slater-Koster coefficients for the differrent boron-boron

TABLE II. The on-site energies of the boron atoms for the 6B: $P 6 \mathrm{mmm}$ structure in units of $\mathrm{eV}$.

\begin{tabular}{ccccc}
\hline \hline & $s$ & $p_{x}$ & $p_{y}$ & $p_{z}$ \\
\hline B & -3.130 & 5.033 & 4.713 & 1.797 \\
\hline \hline
\end{tabular}

bonds are given in Table I and the on-site energies for the boron atoms in Table II. The full Hamiltonian and overlap matrices are given in Supplemental Material [55].

Using Eq. (4) with the coefficients presented in Tables I and II, one obtains the band structure shown in Fig. 4, which is compared to the band energies calculated by DFT. We made sure that the band structure is most accurately reproduced for energies in a wide range around the Fermi energy. The tightbinding model can explain the band-structure calculated by DFT below the Fermi level, because the formalism is based on filled orbitals and the core electrons. The presented model provides us with a good fitting in any $k$ point in the whole Brillouin zone below the Fermi level.

\section{EFFECTIVE LOW-ENERGY HAMILTONIAN}

The hexagonal Brillouin zone of 6B:P6mmm has two inequivalent corners $K$ and $K^{\prime}$. The four branches of its electronic spectrum form two Dirac cones at each $K$ point at different energies (see blue and red circles in Fig. 3). Figure 5 shows the contour plot of the conduction and valence bands near the $K$ point. To recover the nodal line it is necessary to include the trigonal warping. As is apparent from Fig. 5 the conduction and valence bands have significant but opposite trigonal warping. To model the low-energy spectrum around

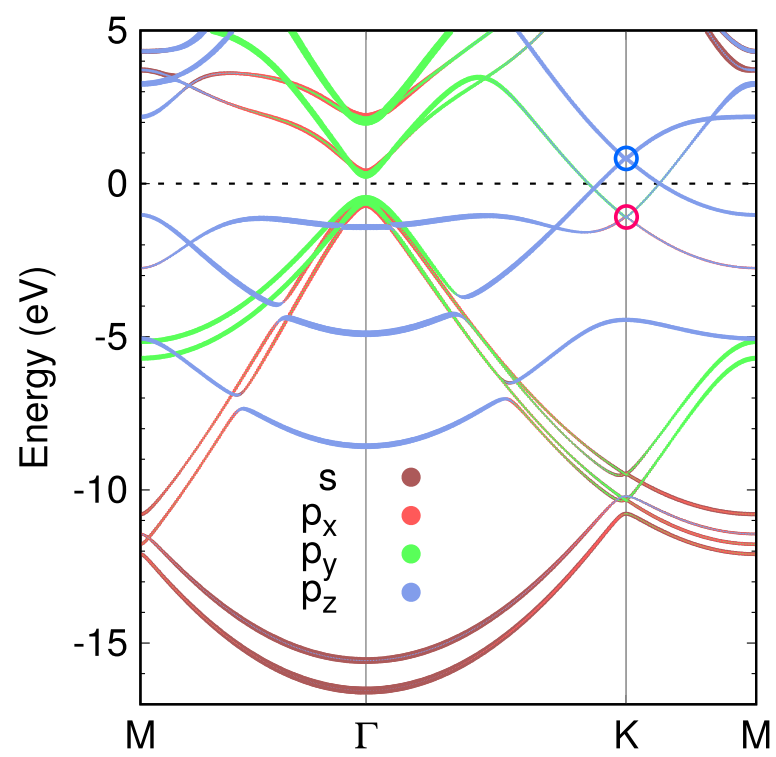

FIG. 3. The band structure of 6B:P6 $6 \mathrm{~mm}$ showing the contribution of different orbitals by the different colors. Blue and red circles show the Dirac cones, which are related to the electrons and holes, respectively. 
the $K$ point, we use an effective mass and group velocity dependent Hamiltonian model. Using unitary transformation, the effective $4 \times 4$ model Hamiltonian near the $K$ point can be expressed in the following form [56-58]:

$$
H=\left(\begin{array}{cccc}
\lambda & -\frac{\Pi^{\dagger^{2}}}{2 m_{e}^{*}}+v_{e} \Pi & V & 0 \\
-\frac{\Pi^{2}}{2 m_{e}^{*}}+v_{e} \Pi^{\dagger} & \lambda & 0 & V \\
V & 0 & -\lambda & -\frac{\Pi^{\dagger^{2}}}{2 m_{h}^{*}}+v_{h} \Pi \\
0 & V & -\frac{\Pi^{2}}{2 m_{h}^{*}}+v_{h} \Pi^{\dagger} & -\lambda
\end{array}\right)
$$

where $\Pi=p_{x}+i p_{y} . m_{e(h)}, V$, and $v_{e(h)}$ are, respectively, the effective mass, perpendicular electric field, and the group velocity of electrons and holes related to conduction and valence bands. The nodal line is formed by the intersection of two Dirac cones, which are located above and below the Fermi level. The Dirac cones are related to the top and bottom honeycomb lattice layers and the energy shift between them is $2 \lambda$. As is evident from Eq. (5) the block related to electrons is shifted by $\lambda$ and for holes it is shifted by $-\lambda$. The dot-dashed line in Fig. 4 shows the bands calculated by the four-band model. The energy bands and the velocities calculated by the low-energy Hamiltonian are more accurate near the Fermi level but start to deviate from the DFT results further away from the Fermi energy, and it is noticeable that its validity is restricted to a region close to the $K$ point and for small

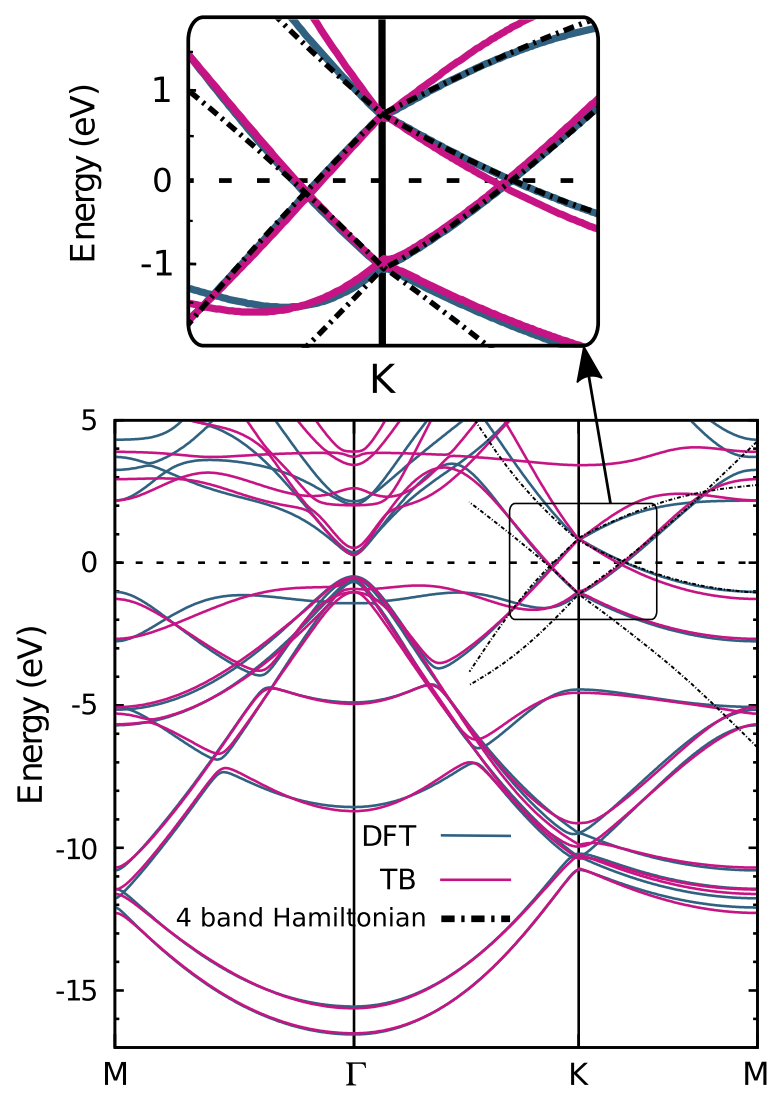

FIG. 4. The fitted band structure of the TB model compared with the one obtained from DFT. The dot-dashed line shows the bands calculated by the low-energy Hamiltonian. energies. One can use the time-reversal operator to jump from the $K$ point to the $K^{\prime}$ point and generate the Hamiltonian around $K^{\prime}$. The low-energy bands near the point $K$ are reproduced by taking $m_{e}=0.174 \hbar^{2} \AA^{-2} \mathrm{eV}^{-1}=1.325 m_{0}$, $m_{h}=0.255 \hbar^{2} \AA^{-2} \mathrm{eV}^{-1}=1.942 m_{0}, \quad \hbar v_{e}=4.744 \mathrm{eV} \AA$ $\hbar v_{h}=6.184 \mathrm{eV} \AA$, and $\gamma=0.992 \mathrm{eV}$. Figure 6 shows a 3D plot of the valence and conduction bands as obtained from the Hamiltonian (5).

By defining $k_{x}=k \cos \varphi$ and $k_{y}=k \sin \varphi$ we find the eigenvalues for the case $V=0$ :

$$
\begin{gathered}
E_{c}=\gamma-\frac{\hbar k}{2 m_{e}} \sqrt{\hbar^{2} k^{2}+4 \hbar k m_{e} v_{e} \cos (3 \varphi)+4 m_{e}^{2} v_{e}^{2}}, \\
E_{v}=-\gamma+\frac{\hbar k}{2 m_{h}} \sqrt{\hbar^{2} k^{2}-4 \hbar k m_{h} v_{h} \cos (3 \varphi)+4 m_{h}^{2} v_{h}^{2}} .
\end{gathered}
$$

The intersection between the two bands defines the equation for the nodal line. After some simplifications we obtain the nodal line $k$ as a function of $\varphi$ :

$$
\hbar k(\varphi)=\frac{4 \gamma}{v_{e}+v_{h}+\sqrt{4 \gamma \cos (3 \varphi)\left(m_{h}^{-1}-m_{e}^{-1}\right)+\left(v_{e}+v_{h}\right)^{2}}} .
$$

This nodal line is presented in Fig. 6 by the red curve. The difference between maximum and minimum energy of the

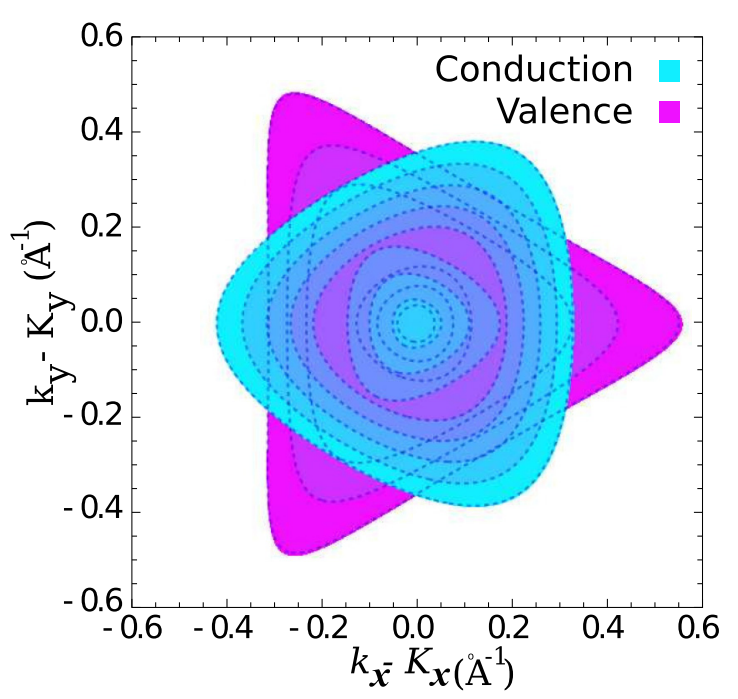

FIG. 5. Contour plot of conduction and valence bands around the $K$ point. 


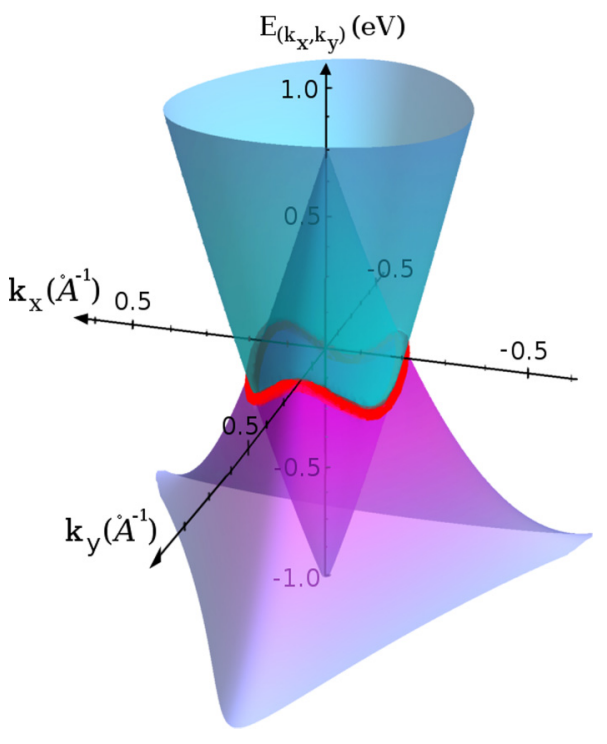

FIG. 6. Valence and conduction bands. The solid red curve around the $K$ point shows the nodal line.

nodal line is $0.18 \mathrm{eV}$. Also in $\beta 12$ borophene there are two Dirac cones and a nodal line, which, however, has a $2.2 \mathrm{eV}$ dispersion in energy near the Fermi level, much larger than in our case [59]. Note that the different trigonal warping for valence and conduction bands is responsible for the breaking of the isoenergetic line and leads to the formation of a nonflat nodal line with dispersion in energy.

One can find the velocities of electrons in conduction and valence band at the nodal line by taking, respectively, the derivatives of Eqs. (6) and (7) with respect to $k$. The equation gives us two different velocities, which are related to the electrons inside (i.e., holes) and outside (i.e., electrons) the nodal line.

Figure 7 shows the velocities of electrons in 6B: $P 6 \mathrm{mmm}$ and graphene as a function of $\varphi$, which reveals that the velocity of electrons is comparable to that of graphene. Note the difference between the position of the minima and maxima of the two velocities, which is related to the difference in the trigonal warping directions shown in Fig. 5.

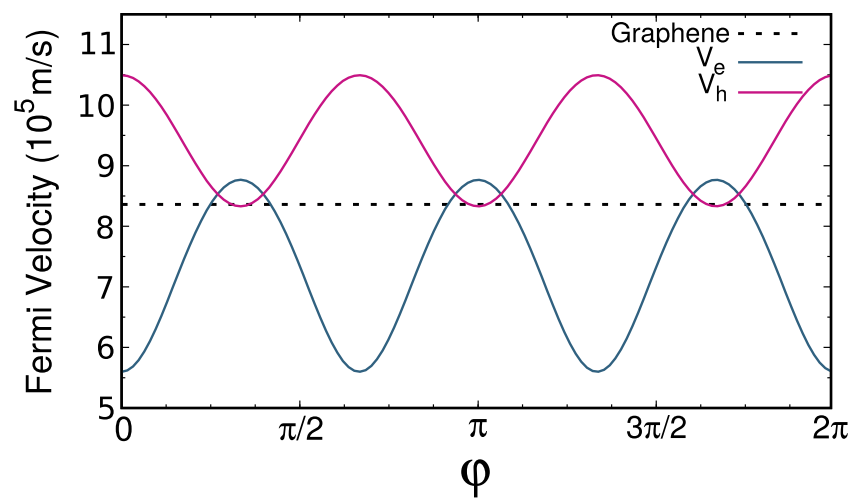

FIG. 7. The Fermi velocity of the electrons (blue) and holes (red) at the nodal line. The dashed black horizontal line represents the velocity of electrons in graphene near the Fermi level.
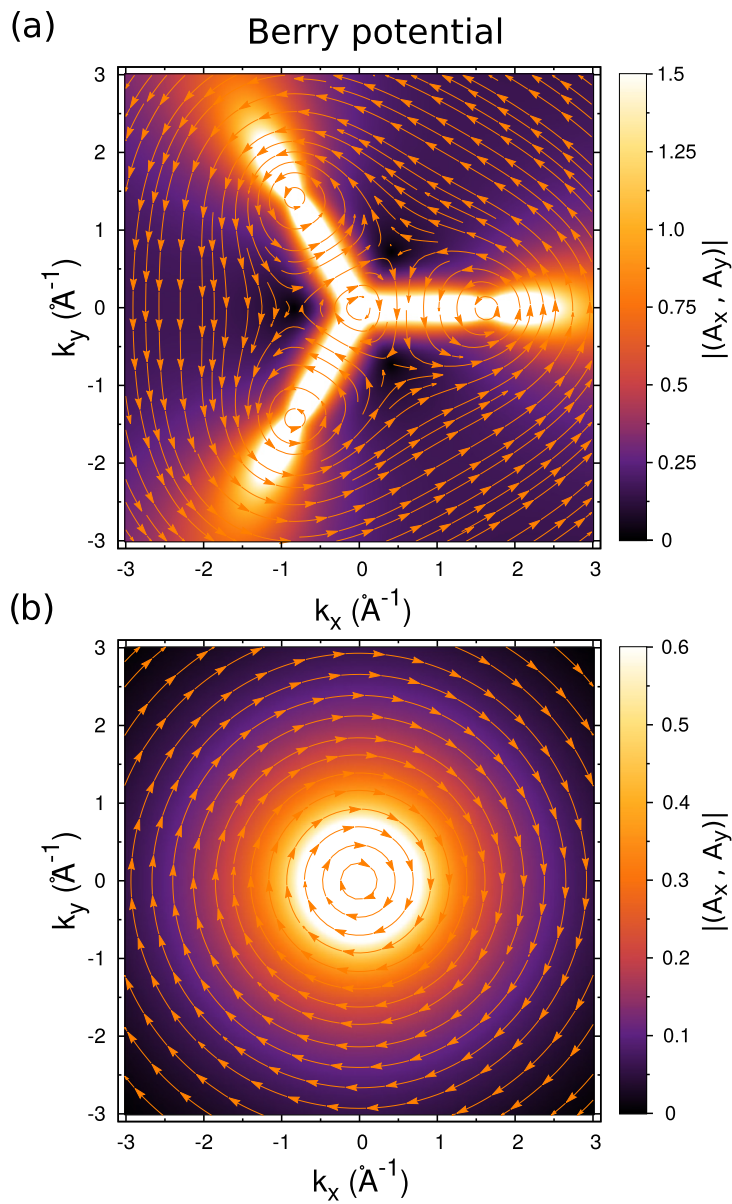

FIG. 8. The Berry potential of the valence band for $6 \mathrm{~B}: P 6 \mathrm{mmm}$ around the $K$ point (a) and for the massless Dirac Hamiltonian (b). Stream lines show the direction of the Berry vector field and the colors indicates the magnitude of the potential vector.

\section{THE EFFECT OF BREAKING SYMMETRIES}

Topologically nontrivial states of crystals have attracted a lot of interest. Of particular interest are Dirac nodal line semimetals (DNLS) and Weyl nodal line semimetals (WNLS) [60]. The 6B:P6mmm structure as a DNLS may be considered as a starting point for generating many other topological states. They can be identified by their Chern number, which is a topological invariant preserved under homeomorphism. Berry phase in quantum mechanics is a geometric phase that is connected to the concept of Chern number. The Berry phase ascribes a phase factor to every path in reciprocal space. The closed-path Berry phase can be physically expressed for each energy band as follows:

$$
\gamma_{n}=\oint_{\mathcal{C}} d \mathbf{k} \cdot \mathcal{A}_{n}(\mathbf{k})
$$

where

$$
\mathcal{A}_{n}(\mathbf{k})=i\left\langle n, \mathbf{k}\left|\nabla_{\mathbf{k}}\right| n, \mathbf{k}\right\rangle
$$

is a singular vector field known as the Berry potential, which is gauge dependent. To determine the Chern number of a given band, we integrate the Berry potential over a closed path, and the Chern number is then given by $C_{n}=\gamma_{n} / 2 \pi$. 


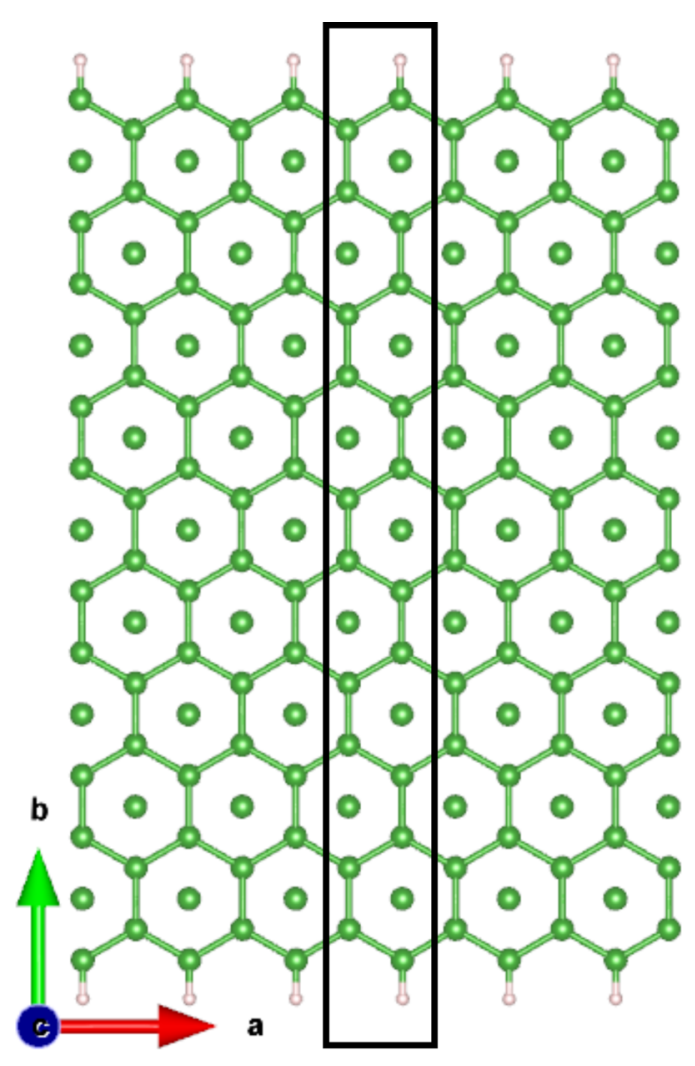

FIG. 9. A zigzag 6B: $86 \mathrm{mmm}$ nano ribbon. The black box shows the unit cell of the ribbon.

For the low-energy band we have the eigenstates analytically, and we calculate the gradient of them and find the Berry potential. Figure 8(a) shows the Berry potential calculated for the valence band around the $K$ point. The color gives the magnitude of the vector field while the arrows indicate the direction. Vortices in the plot reveal the singularities in the vector field. After integrating over a closed path we found the Chern number for every band below the Fermi level to be $C_{n}=1$. After summation over the filled bands we found Chern number $C=2$. This is very different from the massless Dirac Hamiltonian [see Fig. 8(b)] which has $C=0$ or the massive Dirac Hamiltonian with a fractional Chern number $C=1 / 2$.

To show the robustness of the nodal line we investigate how breaking inversion or time reversal leads to a splitting of the nodal line, which does not open a gap. Tje Zeeman field as a real gauge separates the nodal line, and the bands will touch and do not cross each other. Despite the splitting of the nodal line, the system will be gapless. Because the nodal line in this system is not flat and has dispersion in energy, we do not find any forbidden energy level.

Also, we investigated whether pseudo gauge fields can open a gap or not. We found that uniform uniaxial strain could not open any gap or split the nodal line, and only moves the position of the nodal line. Also, nonuniform strain, which is a good candidate for gap engineering, can not split the nodal line. Spin orbit coupling (SOC) in our system does not play a significant role because boron is a light atom. Inducing SOC, we found only a splitting of $0.8 \mathrm{meV}$.
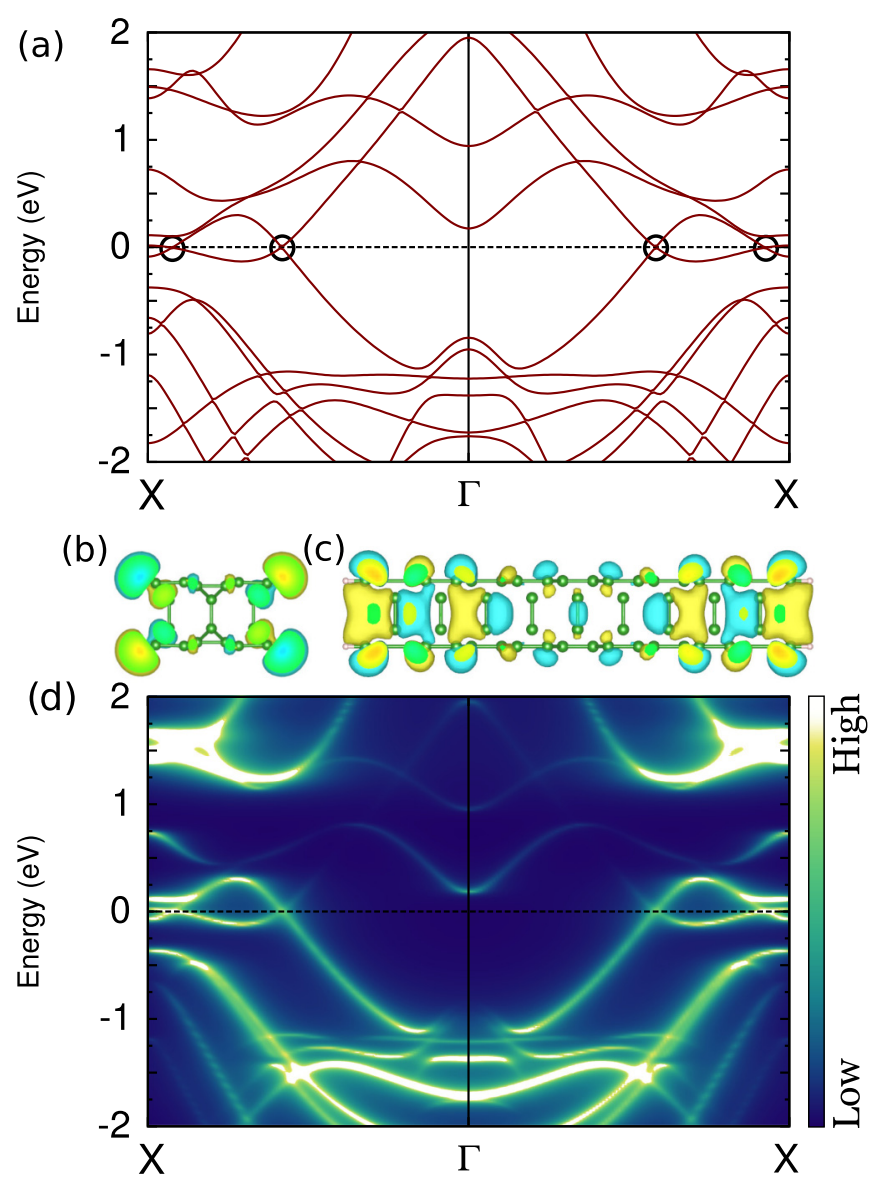

FIG. 10. (a) The band structure of a zigzag 6B: $86 \mathrm{mmm}$ nanoribbon along $X \Gamma X$. Red color indicates the contribution of the $p_{z}$ orbitals of edge atoms. The Bloch function for the nanoribbon including 22 atoms (b) and 62 atoms (c) at the crossing points of the band energies at zero energy. (d) The color bar denotes the weight of the outermost atoms at both edges of the bands.

In light of recent interest in topological states, it is of interest to make a comparison between the sheet and its ribbon and look for the origin of the nodal line. Figure 9 illustrates the zigzag nanoribbon. We study two nanoribbons with 22 and 62 atoms in the unit cell. The edges of ribbons are passivated with hydrogen atoms. Figure 10(a) shows the band structure of the nanoribbon, including 22 atoms, along the path between $\Gamma$ and $X$. The marked cross points with zero energy are equivalent to the nodal line of our $2 \mathrm{D}$ sheet. The eigenstates $\left[\Psi_{n, \mathbf{k}}(\mathbf{R})\right]$ for the crossing bands are presented in Figs. 10(b) and 10(c) for the nanoribbon, including 22 and 62 atoms, respectively. The contribution of the edge states is shown by the color coding in Fig. 10(d). The covalent bonds between borophene layers cause a strong in-plane interaction and consequently the edge states are mixed with the bulk states, which can also be seen in Figs. 10(b) and 10(c). Notice that these states are edge states and they play an important role in forming the nodal line. A similar situation was recently found in the flat semimetal $\mathrm{B}_{2} \mathrm{C}$ [61]. Although there is no proof of the relation between edge states and Chern number, it is widely accepted that the Chern number is equivalent to the number of gapless edge states. As shown in Fig. 10(d), 
there are two gapless edge states in the band structure of bilayer borophene which cross at the Fermi level.

\section{SUMMARY}

To obtain the electronic band structure of bilayer hexagonal borophene 6B: $P 6 \mathrm{mmm}$ we applied density functional theory. In contrast to graphene, which exhibits a Dirac point, we found a nodal line, which is the most dominant characteristic of the band structure of 6B:P6mmm as a topological semimetal. 6B:P6mmm exhibits high-speed Dirac electrons whose velocity is comparable to the Fermi velocity of electrons in graphene.

Based on the Slater-Koster approach, a tight-binding model was constructed that gives a close fit to the DFT results. In order to describe the nodal line, we constructed an effective $4 \times 4$ low-energy model Hamiltonian near the $K$ point and presented an analytical equation for the nodal line as a function of azimuthal angle around the center of the nodal line. The nodal line in the spectrum is a result of two interpenetrating Dirac cones. Due to the different trigonal warping of the electron and hole Dirac cones, the nodal line exhibits an energy dispersion of $0.18 \mathrm{eV}$.

We investigated the origin of the nodal line and found that the edge states play an important role in the formation of the nodal line. Breaking time reversal and inversion symmetries can split the nodal line, but cannot open a gap. The nodal line is also very robust against perturbations and the presence of impurities.

\section{ACKNOWLEDGMENTS}

This work was supported by the Methusalem program of the Flemish government and the graphene FLAG-ERA project TRANS-2D-TMD.
[1] Z. Wang, Y. Sun, X. Q. Chen, C. Franchini, G. Xu, H. Weng, X. Dai, and Z. Fang, Phys. Rev. B 85, 195320 (2012).

[2] Z. Wang, H. Weng, Q. Wu, X. Dai, and Z. Fang, Phys. Rev. B 88, 125427 (2013).

[3] H. B. Nielsen and M. Ninomiya, Phys. Lett. B 130, 389 (1983).

[4] L. Balents, Physics 4, 36 (2011).

[5] X. Wan, A. M. Turner, A. Vishwanath, and S. Y. Savrasov, Phys. Rev. B 83, 205101 (2011).

[6] G. Xu, H. Weng, Z. Wang, X. Dai, and Z. Fang, Phys. Rev. Lett. 107, 186806 (2011).

[7] C. Fang, Y. Chen, H. Y. Kee, and L. Fu, Phys. Rev. B 92, 081201 (2015).

[8] A. A. Burkov, M. D. Hook, and L. Balents, Phys. Rev. B 84, 235126 (2011).

[9] H. Weng, Y. Liang, Q. Xu, R. Yu, Z. Fang, X. Dai, and Y. Kawazoe, Phys. Rev. B 92, 045108 (2015).

[10] J. T. Wang, H. Weng, S. Nie, Z. Fang, Y. Kawazoe, and C. Chen, Phys. Rev. Lett. 116, 195501 (2016).

[11] R. Li, H. Ma, X. Cheng, S. Wang, D. Li, Z. Zhang, Y. Li, and X. Q. Chen, Phys. Rev. Lett. 117, 096401 (2016).

[12] J. L. Lu, W. Luo, X. Y. Li, S. Q. Yang, J. X. Cao, X. G. Gong, and H. J. Xiang, Chin. Phys. Lett. 34, 057302 (2017).

[13] J. Zhao, R. Yu, H. Weng, and Z. Fang, Phys. Rev. B 94, 195104 (2016).

[14] K. Mullen, B. Uchoa, and D. T. Glatzhofer, Phys. Rev. Lett. 115, 026403 (2015).

[15] M. Ezawa, Phys. Rev. Lett. 116, 127202 (2016).

[16] N. B. Kopnin, T. T. Heikkila, and G. E. Volovik, Phys. Rev. B 83, 220503 (2011).

[17] G. E. Volovik, Phys. Scr. T 164, 014014 (2015).

[18] R. Yu, H. Weng, Z. Fang, X. Dai, and X. Hu, Phys. Rev. Lett. 115, 036807 (2015).

[19] B. Feng, B. Fu, S. Kasamatsu, S. Ito, P. Cheng, C. C. Liu, Y. Feng, S. Wu, S. K. Mahatha, P. Sheverdyaeva, and P. Moras, Nat. Commun. 8, 1007 (2017).

[20] Q. Xu, R. Yu, Z. Fang, X. Dai, and H. Weng, Phys. Rev. B 95, 045136 (2017).

[21] L. S. Xie, L. M. Schoop, E. M. Seibel, Q. D. Gibson, W. Xie, and R. J. Cava, APL Mater. 3, 83602 (2015).
[22] G. Bian, T. R. Chang, H. Zheng, S. Velury, S. Y. Xu, T. Neupert, C. K. Chiu, S. M. Huang, D. S. Sanchez, I. Belopolski, N. Alidoust, P. J. Chen, G. Chang, A. Bansil, H. T. Jeng, H. Lin, and M. Z. Hasan, Phys. Rev. B 93, 121113 (2016).

[23] Z. Yan, P. W. Huang, and Z. Wang, Phys. Rev. B 93, 085138 (2016).

[24] J. W. Rhim and Y. B. Kim, Phys. Rev. B 92, 045126 (2015).

[25] S. M. Huang, S. Y. Xu, I. Belopolski, C. C. Lee, G. Chang, B. Wang, N. Alidoust, G. Bian, M. Neupane, C. Zhang, S. Jia, A. Bansil, H. Lin, and M. Z. Hasan, Nat. Commun. 6, 7373 (2015).

[26] B. Q. Lv, H. M. Weng, B. B. Fu, X. P. Wang, H. Miao, J. Ma, P. Richard, X. C. Huang, L. X. Zhao, G. F. Chen, Z. Fang, X. Dai, T. Qian, and H. Ding, Phys. Rev. X 5, 031013 (2015).

[27] X. Huang, L. Zhao, Y. Long, P. Wang, D. Chen, Z. Yang, H. Liang, M. Xue, H. Weng, Z. Fang, X. Dai, and G. Chen, Phys. Rev. X 5, 031023 (2015).

[28] B. Q. Lv, N. Xu, H. M. Weng, J. Z. Ma, P. Richard, X. C. Huang, L. X. Zhao, G. F. Chen, C. E. Matt, F. Bisti, V. N. Strocov, J. Mesot, Z. Fang, X. Dai, T. Qian, M. Shi, and H. Ding, Nat. Phys. 11, 724 (2015).

[29] R. Yu, Z. Fang, X. Dai, and H. Weng, Front. Phys. 12, 127202 (2017).

[30] L. Petersen and P. Hedegård, Surf. Sci. 459, 49 (2000).

[31] Z. K. Liu, B. Zhou, Y. Zhang, Z. J. Wang, H. M. Weng, D. Prabhakaran, S. K. Mo, Z. X. Shen, Z. Fang, X. Dai, and Z. Hussain, Science 343, 864 (2014).

[32] A. J. Mannix, X.-F. Zhou, B. Kiraly, J. D. Wood, D. Alducin, B. D. Myers, X. Liu, B. L. Fisher, U. Santiago, J. R. Guest, M. J. Yacaman, A. Ponce, A. R. Oganov, M. C. Hersam, and N. P. Guisinger, Science 350, 1513 (2015).

[33] B. Feng, J. Zhang, Q. Zhong, W. Li, S. Li, H. Li, P. Cheng, S. Meng, L. Chen, and K. Wu, Nat. Chem. 8, 563 (2016).

[34] Z. H. Zhang, A. J. Mannix, Z. L. Hu, B. Kiraly, N. P. Guisinger, M. C. Hersam, and B. I. Yakobson, Nano Lett. 16, 6622 (2016).

[35] Q. Zhong, L. Kong, J. Gou, W. Li, S. Sheng, S. Yang, P. Cheng, H. Li, K. Wu, and L. Chen, Phys. Rev. Mater. 1, 021001 (2017).

[36] Q. Zhong, J. Zhang, P. Cheng, B. Feng, W. Li, S. Sheng, H. Li, S. Meng, L. Chen, and K. J. Wu, J. Phys.: Condens. Matter 29, 095002 (2017). 
[37] B. F. Decker and J. S. Kasper, Acta Crystallogr. 12, 503 (1959).

[38] Z. A. Piazza, H. S. Hu, W. L. Li, Y. F. Zhao, J. Li, and L. S. Wang, Nat. Commun. 5, 3113 (2014).

[39] X. F. Zhou, X. Dong, A. R. Oganov, Q. Zhu, Y. Tian, and H. T. Wang, Phys. Rev. Lett. 112, 085502 (2014).

[40] A. R. Oganov, J. Chen, C. Gatti, Y. Ma, Y. Ma, C. W. Glass, Z. Liu, T. Yu, O. O. Kurakevych, and V. L. Solozhenko, Nature (London) 457, 863 (2009).

[41] V. Bezugly, J. Kunstmann, B. Grundkötter-Stock, T. Frauenheim, T. Niehaus, and G. Cuniberti, ACS Nano 5, 4997 (2011).

[42] W. Huang, A. P. Sergeeva, H. J. Zhai, B. B. Averkiev, L. S. Wang, and A. I. Boldyrev, Nat. Chem. 2, 202 (2010).

[43] F. Ma, Y. Jiao, G. Gao, Y. Gu, A. Bilic, Z. Chen, and A. Du, Nano Lett. 16, 3022 (2016).

[44] J. C. Slater and G. F. Koster, Phys. Rev. 94, 1498 (1954).

[45] S. Boker, M. Neale, H. Maes, M. Wilde, M. Spiegel, T. Brick, J. Spies, R. Estabrook, S. Kenny, T. Bates, P. Mehta, and J. Fox, Psychometrika 76, 306 (2011).

[46] R. W. Jansen and O. F. Sankey, Phys. Rev. B 36, 6520 (1987).

[47] J. P. Perdew, K. Burke, and M. Ernzerhof, Phys. Rev. Lett. 77, 3865 (1996).

[48] A. D. Becke, Phys. Rev. A 38, 3098 (1988).
[49] W. C. Lu, C. Z. Wang, K. Ruedenberg, and K. M. Ho, Phys. Rev. B 72, 205123 (2005).

[50] C. M. Goringe, D. R. Bowler, and E. Hernandez, Rep. Prog. Phys. 60, 1447 (1997).

[51] W. Kutzelnigg, J. Mol. Struct. THEOCHEM 169, 403 (1988).

[52] S. D. Deshpande and R. B. Pode, Am. J. Phys. 56, 362 (1988).

[53] D. A. Papaconstantopoulos, Handbook of the Band Structure of Elemental Solids (Plenum, New York, 1986).

[54] K. Levenberg, Q. Appl. Math. 2, 164 (1944).

[55] See Supplemental Material at http://link.aps.org/supplemental/ 10.1103/PhysRevB.98.115413 for the Hamiltonian and the overlap matrix in terms of hoppings between different orbitals for bilayer borophene 6B:P6mmm .

[56] E. McCann and V. I. Fal'ko, Phys. Rev. Lett. 96, 086805 (2006).

[57] K. Kechedzhi, V. I. Fal'ko, E. McCann, and B. L. Altshuler, Phys. Rev. Lett. 98, 176806 (2007).

[58] C. Park, J. Ryou, S. Hong, B. G. Sumpter, G. Kim, and M. Yoon, Phys. Rev. Lett. 115, 015502 (2015).

[59] S. Gupta, A. Kutana, and B. I. Yakobson, J. Phys. Chem. Lett. 9, 2757 (2018).

[60] S. Y. Yang, H. Yang, E. Derunova, S. S. Parkin, B. Yan, and M. N. Ali, Adv. Phys.: X 3, 1414631 (2018).

[61] P. Zhou, Z. S. Ma, and L. Z. Sun, arXiv:1710.05144. 